Article

\title{
Bubbly Water as a Natural Metamaterial of Negative Bulk-Modulus
}

\author{
Pi-Gang Luan
}

Department of Optics and Photonics, National Central University, Jhongli District, Taoyuan City 32001, Taiwan; pgluan@dop.ncu.edu.tw

Received: 18 August 2019; Accepted: 30 August 2019; Published: 1 September 2019

\begin{abstract}
In this study, an oscillator model of bubble-in-water is proposed to analyze the effective modulus of low-concentration bubbly water. We show that in a wide range of wave frequency the bubbly water acquires a negative effective modulus, while the effective density of the medium is still positive. These two properties imply the existence of a wide acoustic gap in which the propagation of acoustic waves in this medium is prohibited. The dispersion relation for the acoustic modes in this medium follows Lorentz type dispersion, which is of the same form as that of the phonon-polariton in an ionic crystal. Numerical results of the gap edge frequencies and the dispersion relation in the long-wavelength regime based on this effective theory are consistent with the sonic band results calculated with the plane-wave expansion method (PWEM). Our theory provides a simple mechanism for explaining the long-wavelength behavior of the bubbly water medium. Therefore, phenomena such as the high attenuation rate of sound or acoustic Anderson localization in bubbly water can be understood more intuitively. The effects of damping are also briefly discussed. This effective modulus theory may be generalized and applied to other bubble-in-soft-medium type sonic systems.
\end{abstract}

Keywords: acoustic metamaterial; effective medium; bubble resonance; negative modulus

\section{Introduction}

Metamaterials are usually manmade structures designed to have some exotic properties that are rarely or never seen in natural materials. The split-ring resonators (SRRs) array is a well-studied structure that responds to incident electromagnetic waves in a range of frequencies like a negative-permeability material [1]. Similarly, the structure of periodically arranged rubber-shelled metal spheres embedded in an epoxy background is an acoustic metamaterial with negative mass density for low frequency sound [2]. In both cases, the exotic wave properties are caused by the coupling of the propagating wave in the background medium to the local resonance mechanism in each cell. The negativity of the material parameters in these two cases also implies the existence of a frequency gap [3]. No wave with frequency in the gap can propagate in this metamaterial if it occupies the whole space. If the metamaterial is distributed in a finite region, waves get attenuated before they penetrate through the medium.

Frequency gap and wave attenuation effects can also be achieved using the Bragg scattering [3] mechanism instead of local resonance. The bandgap of a usual photonic [4] or sonic crystal $[5,6]$ is caused by the destructive interference between different 'component waves' in the Fourier series expansion of the Bloch mode. To make such a bandgap large, a high contrast of material parameters between the periodically distributed inclusions and the background material is required [7]. In addition, the center of the gap usually corresponds to a wavelength of about two to three times the lattice constant (spatial period) of the structure. This fact means that in order to isolate waves using non-resonant structures, the entire structure has to be bulky. Therefore, resonant structure is the better choice in this respect. 
Recently, some researchers have noticed that bubble phononic crystals can attenuate sound very effectively, so they designed bubble phononic screens to isolate acoustic waves [8-11]. Similar to the negative-density metamaterial mentioned above, the high attenuation ability of the bubble phononic crystal is caused by the resonance coupling of elastic waves and bubble pulsation. In fact, a similar effect of bubble pulsation resonance in water is a well-known phenomenon and has been studied for decades [12]. The sound attenuation ability of bubbly water has also been discovered theoretically for about two decades $[13,14]$. To the best of our knowledge, the sound attenuation effect has been explained so far by the multiple scattering theory of waves when the bubble positions and bubble sizes are randomly distributed, whereas the concept of phononic bandgap was used if the bubbles were periodically arranged. We will show in this paper that bubbly water can be treated as a natural metamaterial, and the resonance coupling of the pressure wave with the bubble pulsation results in a negative modulus of the medium, giving a low frequency gap.

Acoustic gaps and dispersion effects in a short wavelength regime for sound waves propagating in liquid have also been studied recently and attracted much attention $[15,16]$. The phononic gaps at elevated frequencies emerge due to symmetry breaking in phonon interactions. These theoretical predictions bear a general character shared among soft and biological (non-crystalline) materials/metamaterials, as have been verified through inelastic X-ray scattering (IXS) experiments.

The metamaterial theory of bubbly water we developed in this paper is based on an oscillator model for a pulsating bubble in water. According to this model, the pulsating bubble can be regarded as a radially vibrating oscillator. The spherical bubble (the air region) acts as a spring, and a shell of water around the bubble is the mass connected to the spring. To derive the effective bulk modulus of the bubbly water, it is assumed that all the bubbles have the same size and are periodically distributed in the water background. For the sake of simplicity, we only consider simple cubic (SC) structures. Therefore, a cubic unit cell contains three regions: the air sphere, the mass shell (water), and the water outside the shell. We treat the material in each region as a lump material. We then derive the dynamical modulus by considering the radial oscillator motion and the pressure-volume $(\mathrm{P}-\mathrm{V})$ relation of the air region.

The purpose of this paper is to provide a simple theory to study the acoustic properties of bubbly water at low filling fraction and long wavelengths. The scattering function of a single air bubble in water and the corresponding oscillator model are derived in Section 2. We then use this model to derive the bulk modulus of the bubbly water in Section 3 and briefly discuss the effects of absorption and the possibility of generalizing this theory. Numerical results are presented in Section 4 . Finally, we conclude this paper in Section 5.

\section{Scattering Function and the Oscillator Model for a Single Bubble}

In this section, we derive the acoustic scattering function $f_{s}$ of a small air bubble for the incident plane wave. The resonance frequency $\omega_{0}$ of the radial vibrating motion of the bubble can be read out directly from the scattering function. On the other hand, the radial stiffness constant $C$ of the bubble can be derived by analyze the relation between the inner pressure and the radial displacement of the bubble. We then use the relation $C=m \omega^{2}$ to derive the effective mass $m$ of the oscillator. The result obtained in this section will be used in the next section for constructing the dynamic model we mentioned in the last section.

\subsection{Scattering Function of a Bubble}

Suppose a spherical air bubble of radius $r_{a}$ is surrounded by water, and the pulsation of the bubble is driven by a plane incident wave $p_{i n c}$ of wavelength much larger than the bubble size $\left(k r_{a} \ll 1\right)$; here $k=2 \pi / \lambda$ is the wave number. In this limit the bubble scatters the incident wave isotropically, leading to the scattering wave of the form

$$
p_{\text {scat }}=f_{s} p_{\text {inc }} G(r)
$$


Here, $G(r)=e^{i k r} / r$ represents the spherical wave solution of the Helmholtz equation (with source) $\left(\nabla^{2}+k^{2}\right) G(\boldsymbol{r})=-4 \pi \delta(\boldsymbol{r})$, and $f_{s}$ is the scattering function to be determined later.

Although the bubble is assumed to be small, we also assume that the acoustic process is fast enough to avoid heat conduction between the bubble and the environment, thus the pulsation of the bubble can be approximated as an adiabatic process in the thermodynamics sense, satisfying the adiabatic $P-V$ relation $P V^{\gamma}=$ constant. Here $P$ is the interior pressure of the bubble, $V$ is the bubble volume, and $\gamma=1.4$ is the specific heat ratio for air. Under this assumption, the radial motion of the bubble satisfies

$$
\frac{\Delta P}{P}=-\frac{\gamma \Delta V}{V}=-\frac{3 \gamma \Delta R}{R}
$$

where $\Delta P, \Delta V$, and $\Delta R$ are the variation of the pressure, the volume, and the radius of the bubble, respectively, and $R$ is the instantaneous radius of the bubble, respectively. Denote $\Delta P$ as $p, \Delta R$ as $\xi$, and assume both of them are small comparing to the static value of $P$ and $R$. We can therefore rewrite Equation (2) as

$$
p=-\frac{3 \gamma P_{0}}{r_{a}} \xi=-\frac{3 B_{a}}{r_{a}} \xi
$$

Here $P_{0}$ is the hydrostatic ambient pressure when the incident wave is absent, and $B_{a}=\gamma P_{0}$ is the bulk modulus of the air.

The pressure across the bubble surface must be continuous, therefore $p=p_{\text {inc }}+p_{\text {scat }}$. In addition, both $p$ and $\xi$ get a time factor $e^{-i \omega t}$ under the influence of the incident wave. Differentiate Equation (3) twice with respect to time, we get

$$
\omega^{2}\left(p_{i n c}+p_{s c a t}\right)=\frac{3 \gamma P_{0}}{r_{a}} \ddot{\xi}
$$

However, the radial acceleration $\ddot{\xi}$ is related to the pressure gradient via

$$
\rho_{w} \ddot{\xi}=-\frac{\partial p}{\partial R}=-\frac{\partial p_{\text {scat }}}{\partial R} .
$$

Here $\rho_{w}$ is density of the water, and the second equality is approximately correct under the long wavelength assumption. Combine Equations (1), (4), and (5) and use the fact $k r_{a} \ll 1$, we find

$$
f_{s}=\frac{r_{a}}{\omega_{0}^{2} / \omega^{2}-1-i k r_{a}} .
$$

Here $\omega_{0}$ is the Minnaert frequency, which is the resonance frequency of the 'bubble oscillator', given by

$$
\omega_{0}=\frac{1}{r_{a}} \sqrt{\frac{3 \gamma P_{0}}{\rho_{w}}} .
$$

As can be easily observed, the strongest scattering happens at a frequency very close to the resonance frequency $\omega_{0}$. Therefore, the effective medium properties might be modified by the resonance. We will derive the effective bulk modulus in the next section.

\subsection{The Stiffness Constant and the Mass of the Oscillator Model}

The mechanism of this low frequency resonance can be understood intuitively. When the wavelength is much longer than the bubble radius, the pressure difference across the bubble itself is negligible, thus we can think of the bubble as a perfect spring. However, even under the long wavelength condition, the scattered wave rapidly drops in the radial direction, so there is a pressure difference between the bubble surface and an imaginary "outer surface" that surrounds the bubble and a water shell around the bubble. These two effects together cause the bubble to vibrate radially like 
an oscillator that is driven by a driving force. The bubble itself acts as a spring, the water shell is the mass, and the radial pressure difference is the driving force. The detailed derivation of this oscillator model can be found in [12]. Here we just derive the stiffness constant and the mass of the water shell for later use.

We denote the surface area $4 \pi r_{a}^{2}$ of the bubble as $S_{i}$, and rewrite the bubble pressure $p$ in Equation (3) as $p_{a}$, then according to Equation (3), the radial restoring force $F_{r}=p_{a} S_{i}$ is

$$
F_{r}=-\frac{3 B_{a} S_{i}}{r_{a}} \xi=-C \xi
$$

The radial stiffness constant $C$ is related to the oscillator mass $m$ through the relation $C=m \omega_{0}^{2}$, so we have

$$
m=\frac{3 B_{a} S_{i}}{\omega_{0}^{2} r_{a}}=4 \pi r_{a}^{3} \rho_{w} .
$$

\section{Effective Bulk Modulus and Dispersion Relation}

In this section, we derive the effective bulk modulus of the bubbly water. For the sake of simplicity, we assume all bubbles are of the same size, and their centers are located periodically on the cites of a simple cubic lattice. We denote the bubble volume and the cell volume as $V_{a}$ and $V_{c}$, and the water volume $V_{c}-V_{a}$ in one unit cell as $V_{w}$. The volume filling fraction of the bubbles is denoted as $f$ (do not confuse it with the scattering function $f_{s}$ ). Thus, we have $f=V_{a} / V_{c}$ and $1-f=V_{w} / V_{c}$. We also denote the bulk modulus for air and water as $B_{a}$ and $B_{w}$, respectively. Similar notation rules also apply to the densities $\rho_{a}, \rho_{w}$, the vibration displacements $\boldsymbol{u}_{a}, \boldsymbol{u}_{w}$, and the vibration velocities $\boldsymbol{v}_{a}=\dot{\boldsymbol{u}}_{a}, \boldsymbol{v}_{w}=\dot{\boldsymbol{u}}_{w}$. In addition, the bubble surface is denoted either as $S_{a}$ or $S_{i}$, and the outer surface of the 'mass shell' is denoted as $S_{0}$. Since the volume enclosed by $S_{o}$ is four times of the bubble volume, and the 'outer radius' $r_{o}$ corresponding to $S_{o}$ is related to the bubble radius $r_{a}$ as $r_{0}=2^{2 / 3} r_{a}$. Our goal is to derive the effective density of the medium $\rho_{\text {eff }}$ and the effective bulk modulus $B_{\text {eff }}$ for use in the following two equations

$$
\frac{\partial}{\partial t}\left(\rho_{e f f} \boldsymbol{v}\right)=-\nabla p, \quad \nabla \cdot \boldsymbol{v}=-\frac{\partial}{\partial t}\left(\frac{p}{B_{e f f}}\right) .
$$

The left equation is Newton's second law expressed in density form. Here $\rho_{e f f} v$ is the effective momentum density of the vibrating medium, and $-\nabla p$ plays the role of the force density. Any component of $\nabla p$ is calculated from the pressure difference between the corresponding two boundaries of the cell, divided by the lattice constant. The right equation is in fact the time derivative of the equation $p=-B_{\text {eff }} \nabla \cdot \boldsymbol{u}$, which describes the relation between the volume variation rate $\frac{\Delta V_{c}}{V_{c}}=\nabla \cdot \boldsymbol{u}$ and the corresponding pressure variation $p$ (see Figure 1). With these two equations we can derive the wave equation for monochromatic (single frequency) waves in this sound-bubble coupling system and the dispersion relations of the propagating modes. The results provide simple explanation of some dramatic wave phenomena such as the very high attenuation rate of sound and Anderson localization, both of which have not yet been explained using the simple concept of frequency-dependent effective bulk modulus. 


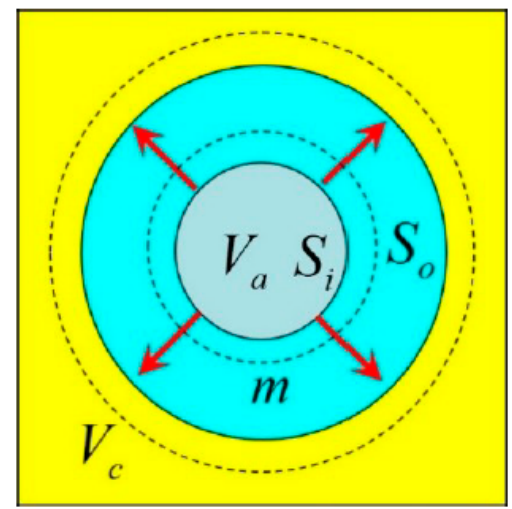

Figure 1. Oscillator model for deriving the effective bulk modulus and effective density. The meaning of the notations are explained in Section 3.

\subsection{The Pressure-Volume Relations and the Effective Bulk Modulus}

We begin with the following relation about the volumes:

$$
\frac{\Delta V_{c}}{V_{c}}=\frac{\Delta V_{a}+\Delta V_{w}}{V_{c}}=f \frac{\Delta V_{a}}{V_{a}}+(1-f) \frac{\Delta V_{w}}{V_{w}} .
$$

Now replace the volume variation rates by the corresponding divergence of the displacements, and apply the relations $\nabla \cdot \boldsymbol{u}=-p / B$, for them, we find

$$
\nabla \cdot \mathbf{v}=-\frac{\partial}{\partial t}\left[f \frac{p_{a}}{B_{a}}+(1-f) \frac{p_{w}}{B_{w}}\right] .
$$

Comparing Equation (12) with Equation (10), and making use of the equality $p_{w}=p$, we get

$$
\frac{1}{B_{e f f}}=\frac{f}{B_{a}}\left(\frac{p_{a}}{p}\right)+\frac{1-f}{B_{w}} .
$$

The ratio $p_{a} / p$ will be derived in the next subsection using the oscillator model.

\subsection{The Dynamics Equation of the Radial Vibrating Oscillator}

We now study the radial motion of the bubble. The mass of the oscillator can be thought of as a water shell of constant density $\rho_{w}$ between the inner surface $S_{i}$ and the outer surface $S_{o}$. The total radial force $p_{a} S_{i}-p S_{o}$ drives the radial motion of the mass shell, satisfying the equation of motion:

$$
p_{a} S_{i}-p S_{o}=m \ddot{\xi}
$$

Applying the sinusoidal condition to $\xi$ ( $\xi$ has a time factor $e^{-\mathrm{i} \omega t}$ ), we get

$$
m \ddot{\xi}=-m \omega^{2} r_{a}\left(\frac{\xi}{r_{a}}\right)=-\frac{m \omega^{2} r_{a}}{3}\left(\frac{\Delta V_{a}}{V_{a}}\right)=\frac{m \omega^{2} r_{a}}{3}\left(\frac{p_{a}}{B_{a}}\right) .
$$

Substitute Equation (15) into Equation (14), we find

$$
\frac{p_{a}}{p}=\frac{3 B_{a} S_{o}}{m r_{a}\left(\omega_{0}^{2}-\omega^{2}\right)} ;
$$

here we have used the alternative expression of the Minnaert frequency

$$
\omega_{0}=\sqrt{\frac{3 B_{a} S_{i}}{m r_{a}}},
$$


which can be derived from Equation (7), Equation (9), and the relation $B_{a}=\gamma P_{0}$.

\subsection{The Effective Bulk Modulus as a Function of Frequency}

Now it is straightforward to derive the frequency-dependent bulk modulus. Substitute Equation (16) into Equation (13), we get

$$
\frac{1}{B_{e f f}}=\frac{1-f}{B_{w}}+\frac{3 f S_{o}}{m r_{a}\left(\omega_{0}^{2}-\omega^{2}\right)}
$$

which can be written as

$$
\frac{1}{B_{e f f}}=\frac{1}{B_{\infty}}\left(\frac{\omega^{2}-\omega_{1}^{2}}{\omega^{2}-\omega_{0}^{2}}\right)=\frac{1}{B_{\infty}}\left(1-\frac{F \omega_{0}^{2}}{\omega^{2}-\omega_{0}^{2}}\right),
$$

where the parameters $B_{\infty}, F$, and $\omega_{1}^{2}$ are given by

$$
B_{\infty}=\frac{B_{w}}{1-f}, \quad F=\frac{\omega_{1}^{2}-\omega_{0}^{2}}{\omega_{0}^{2}}, \quad \omega_{1}^{2}=\omega_{0}^{2}+\frac{3 f}{1-f}\left(\frac{S_{0} B_{w}}{m r_{a}}\right) .
$$

It is clear from Equation (19) that the inverse bulk modulus $B_{e_{f f}}^{-1}$ as a function of frequency has the same form as the dielectric function in the Lorentz model, or that for the polariton wave in the polar medium. According to this observation we learn immediately that the effective bulk modulus for the bubbly water medium becomes negative in the frequency range $\omega_{0}<\omega<\omega_{1}$. This frequency interval is also the frequency gap or forbidden band of the propagating modes.

The bulk modulus of air is given by $B_{a}=\rho_{a} c_{a}^{2}$, where $c_{a}$ is the sound speed in air. Similarly, $B_{w}=\rho_{w} c_{w}^{2}$ gives the bulk modulus of the water. Substitute these two relations and $S_{o} / m=24 / 3 / \rho_{w}=2.52 / \rho_{w}$ into Equation (20), we get

$$
B_{\infty}=\frac{\rho_{w} c_{w}^{2}}{1-f}, \quad F=\frac{2.52 f}{1-f}\left(\frac{\rho_{w} c_{w}^{2}}{\rho_{a} c_{a}^{2}}\right), \quad \omega_{1}=\sqrt{1+F} \omega_{0}
$$

The bulk modulus ratio between water and air is about $1.5 \times 10^{4}$, thus according to Equation (21) even a low filling fraction $f$ will give a large $F$, corresponding to a wide gap. We will derive in the next section the dispersion relation of the propagating modes and discuss the effect of the frequency gap.

\subsection{Dispersion Relation of Acoustic Modes}

To determine the dispersion relation, besides the bulk modulus, we should know the effective mass density. Since we assume the wavelength is long enough, it is reasonable to assume that the bubble moves together (in phase) with the surrounding water in the same cell. This consideration leads us to the simple average result

$$
\rho_{e f f}=f \rho_{a}+(1-f) \rho_{w} .
$$

The correctness of this expression will be tested later.

The wave equation for a monochromatic (single frequency) wave can be derived by substituting Equation (19) and Equation (22) into Equation (10). The result is

$$
\nabla^{2} p-\frac{\rho_{e f f}}{B_{e f f}} \frac{\partial^{2} p}{\partial t^{2}}=0
$$


Here the ratio $\rho_{e f f} / B_{e_{f f}}$ before the double time derivative is the inverse square speed of sound in the bubbly water medium. Adopting water as the standard medium or reference 'wave vacuum', we can define the refractive index of this medium as

$$
n(\omega)=c_{w} \sqrt{\frac{\rho_{e f f}}{B_{e f f}}}=n_{\infty} \sqrt{\frac{\omega^{2}-\omega_{1}^{2}}{\omega^{2}-\omega_{0}^{2}}} .
$$

Here $n_{\infty}=c_{w} \sqrt{\rho_{e f f} / B_{\infty}}$ is a reference index at high frequency. This refractive index becomes imaginary in the frequency gap, which indicates that waves cannot propagate in the medium if its frequency is within the gap.

We can deepen our understanding of the refractive index function by studying the dispersion relation of the propagating modes. Substitute the plane wave form $p=p_{0} e^{i(\boldsymbol{K} \cdot \boldsymbol{r}-\omega t)}$ into Equation (23), we get

$$
K^{2}=\frac{n^{2}(\omega) \omega^{2}}{c_{\omega}^{2}}=\frac{n_{\infty}^{2} \omega^{2}}{C_{\omega}^{2}}\left(\frac{\omega^{2}-\omega_{1}^{2}}{\omega^{2}-\omega_{0}^{2}}\right) .
$$

This dispersion relation is of the same form as that for the phonon-polariton waves. According to Equation (25), propagating modes can exist with frequency below $\omega_{0}$ or above $\omega_{1}$. Within the interval $\omega_{0}<\omega<\omega_{1}$, the wave vector becomes imaginary, thus the wave amplitude decays along the wave vector direction. If the bubbly water medium is filled in a slab region of finite thickness, an incident wave having a frequency within the gap would get attenuated before it penetrates through the slab. Furthermore, if a pulsating source of sound surrounded by the 'bubble cloud' radiates sound waves of frequency in the gap, the sound wave would be trapped by the bubble cloud, leading to the phenomena such like the Anderson localization of acoustic waves.

The negative modulus of the bubbly water is caused by the dynamical coupling of the sound field in water and the local resonance of every bubble oscillator. Similar mechanism for polar crystal also leads to the negative dielectric function in the polariton gap. The main difference between these two systems is that in the bubbly water case the oscillators vibrate radially so they couple with the scalar pressure field, whereas in the polar crystal case the local dipoles (for example, the $\mathrm{Na}^{+}-\mathrm{Cl}^{-}$pairs) are coupled with the electric vector field.

\subsection{Absorption Effect and the Possibility of Generalization}

In the derivation of the effective bulk modulus, we never considered any absorption or loss effects. For real bubbles, the damping effect caused by thermal conductivity (non-adiabatic correction), shear viscosity absorption at the bubble surface, and the radiation loss due to scattering must be considered. These corrections will cause the resonance frequency of the bubble to change slightly, reducing the peak of the scattering function and widening its width. These corrections will also introduce a damping force on the radial motion of the bubble oscillator and change the bulk modulus such that it does not acquire an unphysical infinite value. Since the absorption effect is a very cumbersome effect, and our aim in this study is not to study this effect, we will not further address this issue. We follow the spirit of $[13,14]$ to choose the proper bubble size so that we can avoid the effects of the absorption.

Another question is how to generalize our theory to elastic solid media containing bubbles. As the authors have demonstrated in [8-11], for elastic media with very low shear stress, the primary mechanism of the first band gap is still caused by the resonance of the bubble. Therefore, we believe that our theory can be generalized and applied to such a system without much difficulty.

\section{Numerical Results}

In this section we discuss some numerical results based on our theory. Hereafter we assume $\rho_{a}=1.2 \mathrm{~kg} / \mathrm{m}^{3}, \rho_{w}=1000 \mathrm{~kg} / \mathrm{m}^{3}, c_{a}=343 \mathrm{~m} / \mathrm{s}$, and $c_{w}=1490 \mathrm{~m} / \mathrm{s}$. These parameters give us 
the bulk modulus $B_{a}=\rho_{a} c_{a}^{2}=1.412 \times 10^{5} \mathrm{~Pa}$ and $B_{w}=\rho_{w} c_{w}^{2}=2.22 \times 10^{9} \mathrm{~Pa}$. If the radius of a bubble is measured in $\mathrm{mm}$, the Minnaert frequency measured in $\mathrm{kHz}$ and we get a simple formula $\omega_{0}=20.58 / r_{a}$ or $v_{0}=\omega_{0} / 2 \pi=3.28 / r_{a}$. To avoid high absorption, we assume the bubbles have radius larger than $100 \mu \mathrm{m}[12,13]$.

The scattering function $f_{s}$ as a function of dimensionless frequency $k r_{a}=\omega r_{a} / c_{w}$ is plotted in Figure 2. Here we replaced the $f_{s}$ function by its dimensionless correspondence $f_{s} / r_{a}$ because here the information of the size is irrelevant. According to Figure 1, the absolute value as well as the imaginary part of $f_{s}$ goes to the maximum value $72.5 r_{a}$ at $k r_{a}=0.0138$, and behaves like an even function with respect to this peak location. The real part of $f_{s}$, on the other hand, first raises to $36.6 r_{a}$, and then drops to zero at this place, and continually goes downwards to $-36.5 r_{a}$, then approaches the horizontal axis without crossing the zero value. Resonance of this kind belongs to the Lorentz dispersion, which is responsible for the negative bulk modulus and the low frequency gap of this bubbly water medium.

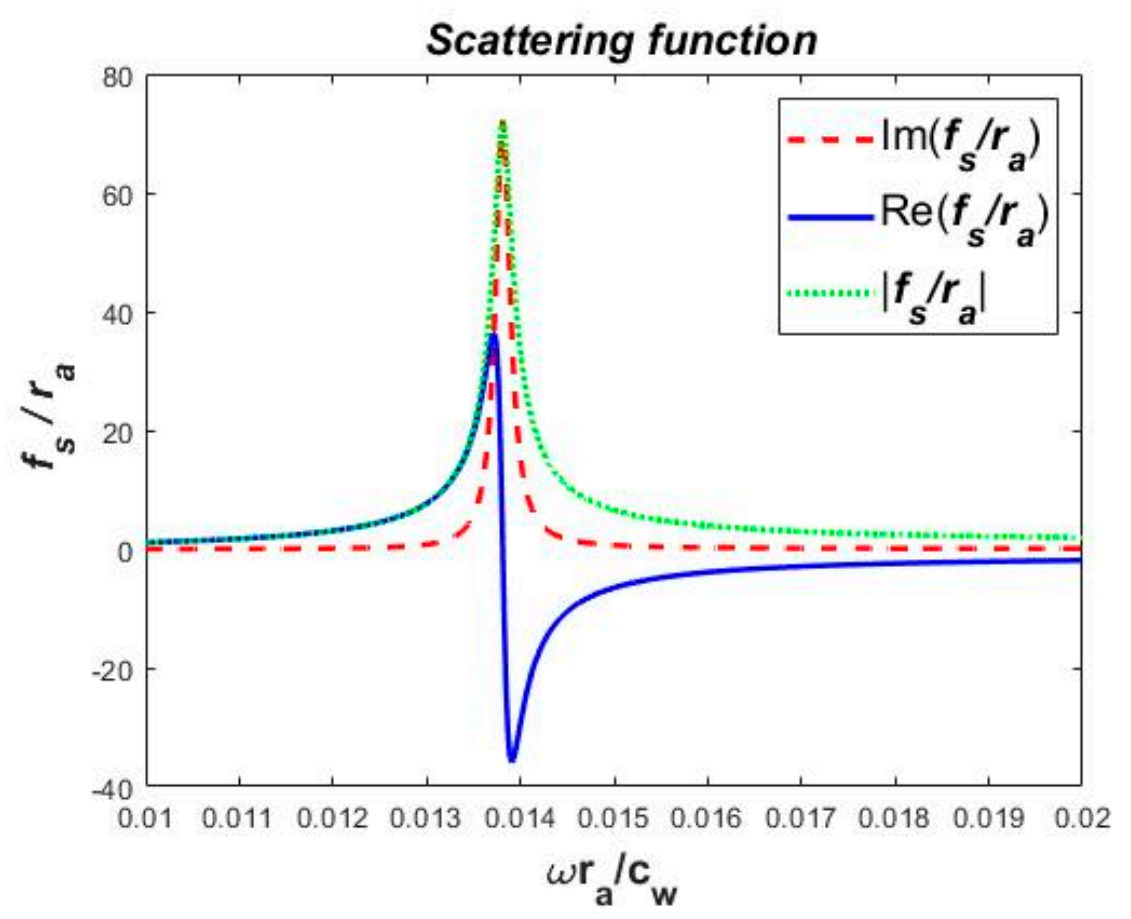

Figure 2. The scattering function of a single bubble. The notations in this figure have been defined in Section 2.

We now study the characteristics of the acoustic band structures of the bubbly-water sonic crystals. Plane wave expansion method (PWEM) were implemented for the calculations. Results for filling fraction $f=0.001$ and $f=0.01$ are shown in Figure 3. These results indicate that bubbly-water sonic crystal is an ideal structure to open large gap at low frequency. For the case with filling fraction $f=0.001$, the gap is opened between $\omega_{L} r_{a} / c_{w}=0.0185$ (at X) and $\omega_{U} r_{a} / c_{w}=0.085$ (at $\Gamma$ ). Comparing them with the gap boundaries $\omega_{0} r_{a} / c_{w}=0.0138$ and $\omega_{1} r_{a} / c_{w}=0.0881$ predicted by the effective metamaterial theory, we find excellent agreement between them. The low frequency limit of the gap in Figure 3 is a little higher than the prediction. We believe this discrepancy is due to the bad convergence of the PWEM at low filling fraction. Similarly for the $f=0.01$ case, we have the gap opened from $\omega_{L} r_{a} / c_{w}=0.0173$ (at X) to $\omega_{U} r_{a} / c_{w}=0.271$ (at $\mathrm{i}$ ). The gap boundaries provided by the effective theory are $\omega_{0} r_{a} / c_{w}=0.0138$ and $\omega_{1} r_{a} / c_{w}=0.277$, also in very good agreement with the band structure result. We noticed that the agreement between $\omega_{L}$ and $\omega_{0}$ in this case is better than in the former case. This fact is consistent with our judgement about the PWEM. 
(a) Acoustic Bands $(f=0.001)$

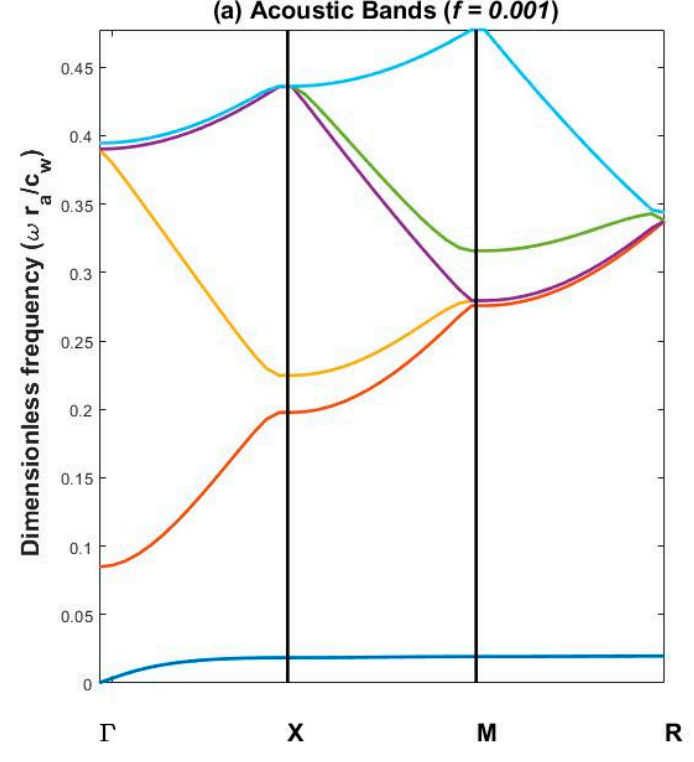

(b) Acoustic Bands $(f=0.01)$

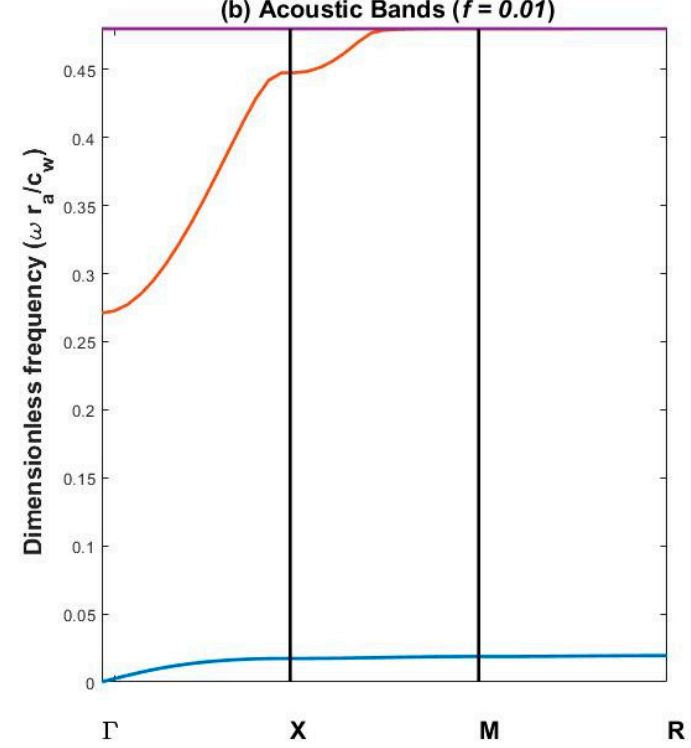

Figure 3. The acoustic band structures for filling fraction (a) $f=0.001$, and $(\mathbf{b}) f=0.01$.

The metamaterial theory developed in this paper not only can provide the range of the gap, but also can give us the attenuation rate of the wave, which is directly related to the imaginary part of the effective wave number that determined by Equation (25). In addition, absorption or loss effect can be easily included by a simple replacement of the frequency: $\omega \rightarrow \omega(1+i \delta)$ [12]. Here $\delta$ is a positive number smaller than 0.1 if we assume the that the bubble radius is larger then $100^{1} \mathrm{~m}$. For the purpose of demonstration, in the simulation of lossy media, we take $\delta=0.05$. Figure $4 \mathrm{a}, \mathrm{b}$ is the results for the lossless media, while Figure $4 \mathrm{c}, \mathrm{d}$ is the results for the lossy media. Here the dimensionless variable $K a$ is the product of the wavenumber $K$ in Equation (25) times the lattice constant $a$ of the SC structure. the lattice constant in the SC structure. The real part of the wave number (wave vector) of the mode waves are represented by the blue solid curves, and the red broken lines represent the imaginary part of the wave vector. These dispersion relations are similar to the dispersion relations of the phonon-polariton that mentioned in the previous section. We noticed that the presence of loss or absorption does not destroy the attenuation effect because it is so large.
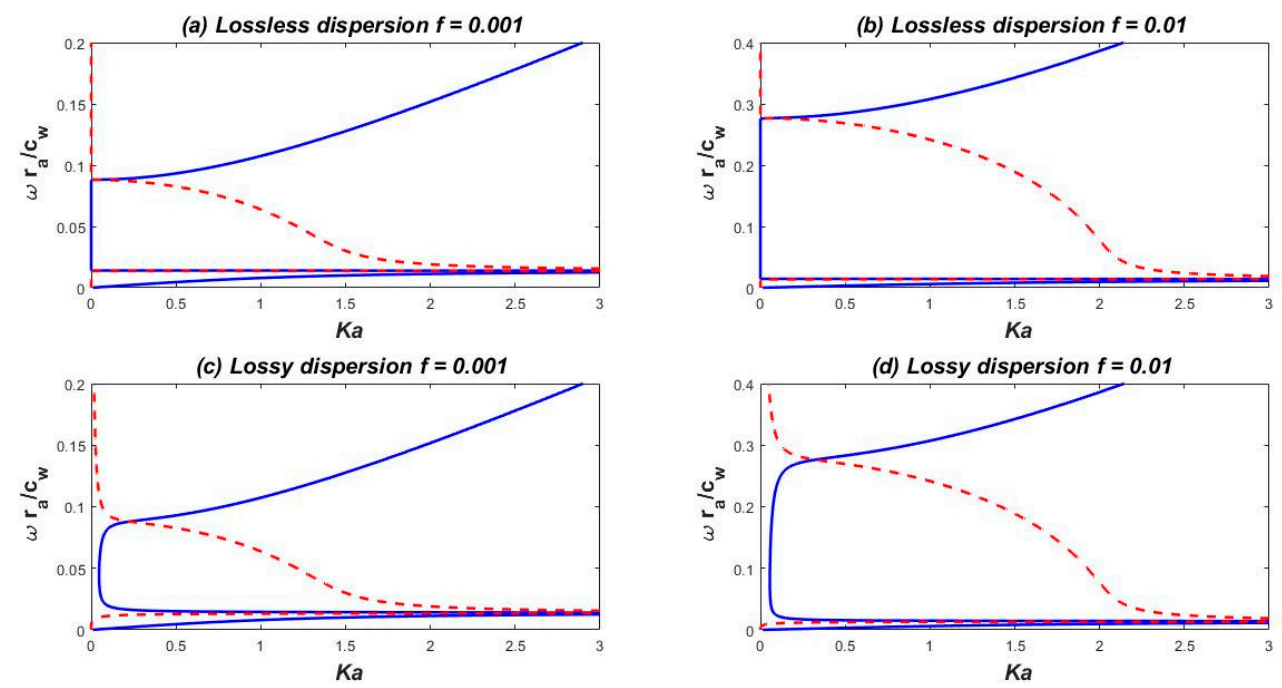

Figure 4. Dispersion relations for $\mathrm{f}=0.001$ and $\mathrm{f}=0.01$, lossless media are shown in (a) and (b). The corresponding results for lossy media are shown in $(\mathbf{c}) f=0.001$ and $(\mathbf{d}) f=0.01$. 
Now we discuss the effective bulk modulus according to Equation (19) and its modified form when loss cannot be ignored. As before, four cases are studied, and their results $\left(B_{\text {eff }} / B_{w}\right)$ are shown in Figure 5. The parameters used in the calculations are the same as those used in Figure 4. The small circles in the four subplots indicate the starting frequencies (changes from positive to negative) of the negative bulk modulus. For the lossless cases, the starting points and the resonance points (infinite effective modulus) are the same as the gap boundaries of the metamaterial. The loss effect seemed not to change the effective bulk modulus much if the frequency is not very close to the resonance point. However, the effective modulus at the resonance frequency no longer goes to the unphysical value of infinity, as expected.
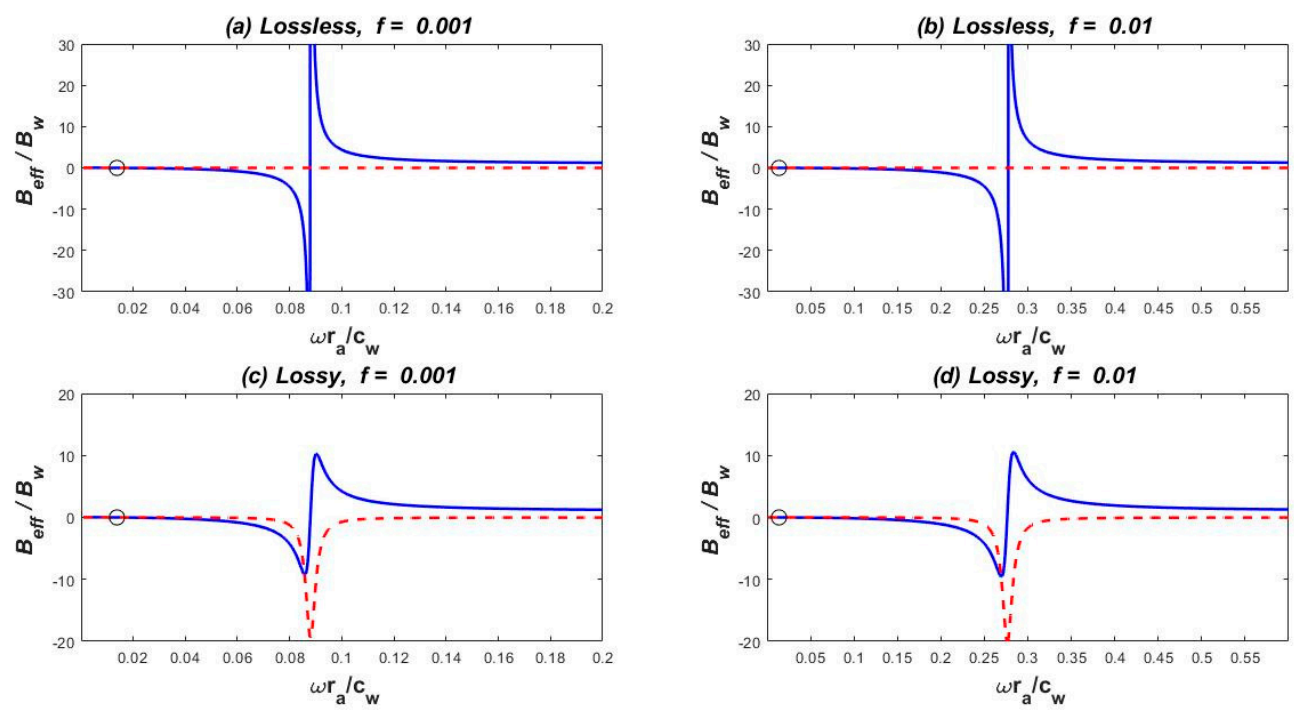

Figure 5. Bulk modulus for lossless cases with $f=0.001$ and $f=0.01$ are shown in (a) and (b).

The corresponding lossy cases are shown in $(\mathbf{c}) \mathrm{f}=0.001$ and $(\mathbf{d}) \mathrm{f}=0.01$.

Finally, we discuss the valid range of the theory developed in this paper. Based on our numerical simulations, we found that the gap range given by the effective theory is in good agreement with that obtained from the band structure calculation (the discrepancy is less than $3 \%$ ) if the filling fraction $f$ of the bubbles is within the range 0.0005 to 0.02 . Beyond this range, we are not sure about the accuracy, and need further investigation. In addition, the bubble radius must not be less than $100 \mu \mathrm{m}$, otherwise high absorption effects must be considered [12].

\section{Conclusions}

In this paper, we apply the oscillator model of air-bubble-in-water to the bubbly water medium and derive the effective bulk modulus of the medium. We show that in a wide range of frequency the effective modulus becomes negative, while the effective density of the medium is still positive. These properties imply the existence of a wide acoustic gap, consistent with band structure calculations. The dispersion relation for the acoustic modes in this medium has Lorentz type dispersion, like that of the phonon-polariton. The theory proposed in this paper provides a simple mechanism for explaining the long-wavelength behavior of the bubbly water medium. Using this theory, phenomena such as the high attenuation rate through a bubble screen or the acoustic Anderson localization in bubbly water can be understood more intuitively. The effects of damping are also discussed. This effective modulus theory may be generalized and applied to other similar structural sonic medium containing periodically arranged bubbles.

Funding: This research received no external funding.

Conflicts of Interest: The author declares no conflict of interest. 


\section{References}

1. Smith, D.R.; Willie, J.P.; Vier, D.C.; Nemat-Nasser, S.C.; Schultz, S. Composite Medium with Simultaneously Negative Permeability and Permittivity. Phys. Rev. Lett. 2000, 84, 4184. [CrossRef] [PubMed]

2. Liu, Z.; Zhang, X.; Mao, Y.; Zhu, Y.Y.; Yang, Z.; Chan, T.C.; Sheng, P. Locally Resonant Sonic Materials. Science 2000, 289, 1734. [CrossRef] [PubMed]

3. Kittel, C. Introduction to Solid State Physics, 8th ed.; John Wiley \&Sons Inc.: Hoboken, NJ, USA, 2005.

4. Joannopoulos, J.; Johnson, S.; Winn, J.; Meade, R. Photonic Crystals: Molding the Flow of Light. Princeton University Press, 2008. Available online: http://ab-initio.mit.edu/book/photonic-crystals-book.pdf (accessed on 31 August 2019).

5. Sánchez-Pérez, J.V.; Caballero, D.; Mártinez-Sala, R.; Rubio, C.; Sánchez-Dehesa, J.; Meseguer, F.; Llinares, J.; Gálvez, F. Sound Attenuation by a Two-Dimensional Array of Rigid Cylinders. Phys. Rev. Lett. 1998, 80, 5325. [CrossRef]

6. Kushwaha, M.S. Stop-bands for periodic metallic rods: Sculptures that can filter the noise. Appl. Phys. Lett. 1997, 70, 3218. [CrossRef]

7. Kushwaha, M.S.; Halevi, P. Giant acoustic stop bands in two-dimensional periodic arrays of liquid cylinders. Appl. Phys. Lett. 1996, 69, 31. [CrossRef]

8. Leroy, V.; Bretagne, A.; Fink, M.; Willaime, H.; Tabeling, P.; Tourin, A. Design and characterization of bubble phononic crystals. Appl. Phys. Lett. 2009, 95, 171904. [CrossRef]

9. Bretagne, A.; Tourin, A.; Leroy, V. Enhanced and reduced transmission of acoustic waves with bubble meta-screens. Appl. Phys. Lett. 2011, 99, 221906. [CrossRef]

10. Leroy, V.; Bretagne, A.; Lanoy, M.; Tourin, A. Band gaps in bubble phononic crystals. AIP Adv. 2016, 6, 121604. [CrossRef]

11. Leroy, V.; Chastrette, N.; Thieury, M.; Lombard, O.; Tourin, A. Acoustics of Bubble Arrays: Role Played by the Dipole Response of Bubbles. Fluids 2018, 3, 95. [CrossRef]

12. Clay, C.S.; Medwin, H. Acoustical Oceanography: Principles and Applications; John Wiley \& Sons: Hoboken, NJ, USA, 1977.

13. Ye, Z.; Hsu, H. Phase transition and acoustic localization in arrays of air bubbles in water. Appl. Phys. Lett. 2001, 79, 1724. [CrossRef]

14. Kafesaki, M.; Penciu, R.S.; Economou, E.N. Air Bubbles in Water: A Strongly Multiple Scattering Medium for Acoustic Waves. Phys. Rev. Lett. 2000, 84, 6050. [CrossRef] [PubMed]

15. Bolmatov, D.; Zhernenkov, M.; Zav'yalov, D.; Stoupin, S.; Cunsolo, A.; Cai, Y.Q. Thermally triggered phononic gaps in liquids at THz scale. Sci. Rep. 2016, 6, 19469. [CrossRef] [PubMed]

16. Bolmatov, D.; Zhernenkov, M.; Zav'yalov, D.; Stoupin, S.; Cai, Y.Q.; Cunsolo, A. Revealing the mechanism of the viscous-to-elastic crossover in liquids. J. Phys. Chem. Lett. 2015, 5, 2785-2790. [CrossRef] [PubMed] 